\title{
A comparative effectiveness study of two culturally competent models of diabetes self-management programming for Latinos from low-income households
}

Janet Page-Reeves ${ }^{*}$ (D) Lidia Regino, Cristina Murray-Krezan, Molly Bleecker, Erik Erhardt, Mark Burge, Elaine Bearer and Shiraz Mishra

\begin{abstract}
Background: Diabetes risk is extremely high for Latinos from low-income households. Health guidelines recommend that individuals learn strategies to self-manage their diabetes, but getting people to adopt required lifestyle changes is challenging and many people are not able to prevent their pre-diabetes from escalating or effectively control their diabetes. Systematic reviews show that culturally competent self-management programs can significantly improve diabetes outcomes and different models for culturally competent programming have been developed.

Methods: This patient-engaged study will compare the effectiveness of two distinct evidence-based models for culturally competent diabetes health promotion at two sites that serve a large Latino patient population from lowincome households: 1) The Diabetes Self-Management Support Empowerment Model, an educational session approach, and 2) The Chronic Care Model, a holistic community-based program. Data collection will involve interviews, focus groups, surveys and assessments of each program; and testing of patient participants for A1c, depression, Body Mass Index (BMI), and chronic stress with hair cortisol levels. We will recruit a total of 240 patient-social support pairs: Patients will be adults (men and women over the age of 18) who: 1.) Enter one of the two diabetes programs during the study; 2.) Self-identify as "Latino;" 3.) Are able to identify a social support person or key member of their social network who also agrees to participate with them; 4.) Are not pregnant (participants who become pregnant during the study will be excluded); and 5.) Have household income 250\% of the Federal Poverty Level (FPL) or below. Social supports will be adults who are identified by the patient participants. PRIMARY OUTCOME: Improved capacity for diabetes selfmanagement measured through improvements in diabetes knowledge and diabetes-related patient activation. SECONDARY OUTCOME: Successful diabetes self-management as measured by improvements in A1c, depression scale scores, BMI, and circulating levels of cortisol to determine chronic stress.
\end{abstract}

Discussion: Our hypothesis is that the program model that interfaces most synergistically with patients' culture and everyday life circumstances will have the best diabetes health outcomes.

Trial registration: This study was registered with ClinicalTrials.gov on December 16, 2016 (Registration \#NCT03004664).

Keywords: Diabetes, Self-care, Cultural competency, Hispanic Americans, Low-income

\footnotetext{
* Correspondence: JPage-Reeves@salud.unm.edu

University of New Mexico Health Sciences Center, Albuquerque, USA
} 


\section{Background}

Although diabetes is a national health crisis, risk is not the same for everybody. Individuals from minority and ethnic populations and those with low-income status are at significantly higher risk [1]. Health guidelines emphasize the importance of patient self-care over narrow reliance on medical treatments for reducing the health impact of diabetes and improving diabetes health outcomes. However, systematic reviews have repeatedly demonstrated that culturally competent health promotion approaches that account for culture and the social context of poverty can be key to improving health outcomes [2-11]. In particular, culturally competent selfmanagement interventions have been shown to significantly improve both glycemic control and behaviors related to diet and physical activity, and also to increase diabetes-related knowledge. A variety of different models have been developed to create "culturally competent" diabetes self-management programs [2-11]. Yet, there is no agreement on what cultural competence specifically means or entails, and, because of a continued emphasis on individual behavior in approaches to diabetes health promotion, the design of self-management programs does not always create cultural competence in a way that makes sense in relation to patients' lives or improve their health.

\section{Methods/Design}

This patient-engaged project compares the effectiveness of two distinct evidence-based models for culturally competent diabetes self-management health promotion currently being implemented by programs available to Latino patients from low-income households in Albuquerque, New Mexico. We hypothesize that the program model that interfaces most synergistically with patient's culture and everyday life circumstances will have the better diabetes health outcomes (See Fig. 1: Conceptual Model).

\section{Aims}

Aim \#1: Characterize the ways that two distinct culturally competent diabetes self-management programs interface with patient culture and socioeconomic context.
Aim \#2: Measure and compare improvement in patient capacity for diabetes self-management.

Aim \#3: Measure and compare patient success at diabetes self-management.

\section{Design}

Data will be gathered at baseline, three, six and 12 months by community data collectors who will be members of the research team. Each of the comparator sites is implementing a program in a "real life" setting, thus providing the opportunity for a pragmatic assessment of the comparative effectiveness of the program models under externally valid and generalizable conditions.

\section{Study population demographics}

New Mexico, one of four majority minority states, has the largest percentage of Latinos in the United States (46.3\% of the state's population) [12]. Of New Mexico's nearly one million Latino residents, most are of Mexican ancestry (62\%) [13]. In Albuquerque, $47 \%$ of the population is Latino [14]. In New Mexico, 18.9\% of Latinos live in households below the poverty level and $12.4 \%$ of Latinos have diabetes [15]. Census data reveal that Mexicanheritage Latinos tend to self-identify as racially "white" (53\%) or as "some other race" (37\%) [16]. This fact and the specific racial make-up of the New Mexico population influence our anticipated enrollment.

Source of Recruitment, Including the Volume of Eligible Patients Available and Expected Participation Rate. We will recruit 240 patient participants from the two programs through our site partner institutions. Estimates of current clinical use suggest that we will be able to successfully recruit participants to meet our target enrollment (See Table 1).

Comparator \#1. The Diabetes Self-Management Support Empowerment Model (DSMS) [17]. The DSMS is a patient-centered, theoretically based educational framework. It combines a series of clinically informed group didactic sessions that use a patient self-determination approach to empower patients to take control of their own diabetes health with follow-up supports to sustain self-management gains achieved during the sessions.

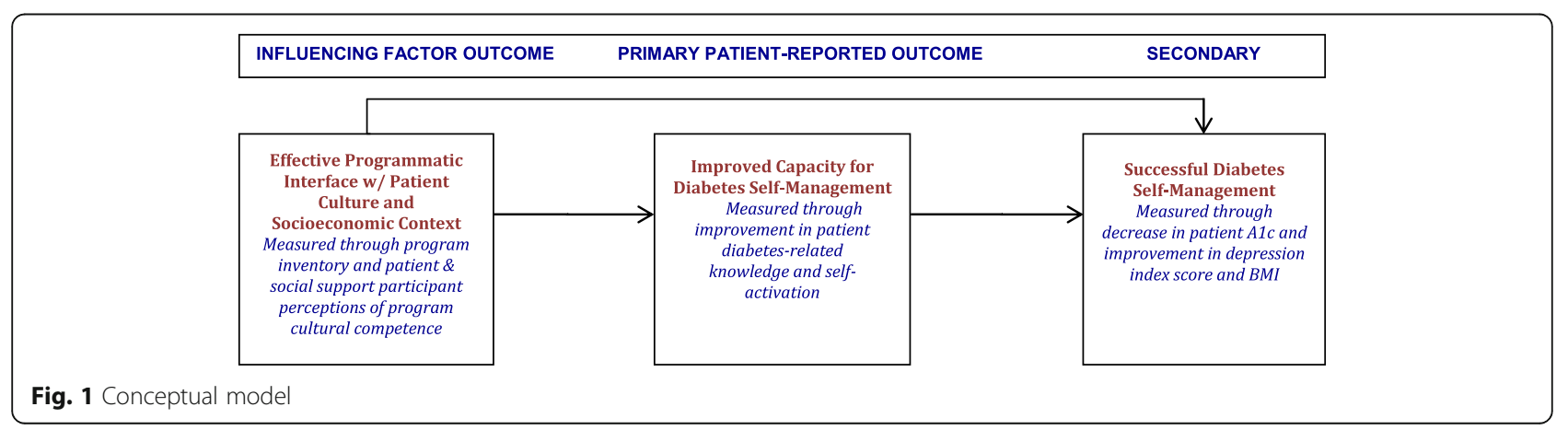


Table 1 Target Enrollment

\begin{tabular}{lllllll}
\hline Program & \# Patients (annually) & \# (\%) latino & $\begin{array}{l}\text { \# (\%) at or below } \\
250 \% \mathrm{FPL}\end{array}$ & \# with diabetes & Annual target enrolment & Total target enrolment \\
\hline CCM & 1032 & $981(95 \%)$ & $1032(100 \%)$ & 142 & 40 Patients and & 120 Patients and \\
& & & & 40 Social supports & 120 Social supports \\
DSMS & 427 & $145(34 \%)$ & $149(35 \%)$ & 427 & 40 Patients and & 120 Patients and \\
& & & & 40 Social supports & 120 Social supports \\
\hline
\end{tabular}

CCM chronic care model

DSMS the diabetes self-management support empowerment model

FPL Federal poverty level

The DSMS requires that educators acquire proficiency in culturally competent supportive care across the lifespan as one of five domains for certification. Educators are expected to be informed about, anticipate, and be aware of specific challenges that might accrue in the patient's diabetes self-management experience.

Comparator \#2. The Chronic Care Model (CCM) [11, 18]. The CCM involves six synergistic domains: 1.) Improved access to care, 2.) Patient self-management support, 3.) Patient decision support, 4.) Care coordination, 5.) Integrated health information systems, and 6.) Access to community resources. To create a holistic care regime, the CCM focuses on addressing social determinants of health by meeting the medical, cultural, and linguistic needs of patients through integration of cultural norms and social relationships from the patient population into the program design.

\section{Participant characteristics}

This project will enroll three types of participants for different components of the study:

- Patients

- Social supports

- Program staff and providers

Patient $(n=240)$ \& social support $(n=240)$ participants Participants for this study will be enrolled as patientsocial support dyads. One hundred twenty patients and their corresponding social supports will be enrolled at each site. All patient participants will be adults (men and women over age 18) who: 1.) have been identified by a provider as having pre-diabetes (A1c between 5.7$6.4 \%$ ) or diabetes (A1c $\geq 6.5 \%)$ and enter one of the two diabetes programs during the study; 2.) self-identify as "Latino" or "Hispanic;" 3.) are able to identify a social support person or key member of their social network who also agrees to participate with them; 4.) are not pregnant (participants who become pregnant during the study will be excluded); and 5.) have household income $250 \%$ of the FPL or below. Social support participants will be adult individuals who are identified by the patient participants.

\section{Subset of patient and social support participants for interviews (72) and focus groups (72)}

At each of the sites, we will identify a purposive sample of interviewees and focus group participants from those already recruited to be in the study and invite them to participate.

\section{Staff \& providers $(n=36)$}

As part of our program assessment, we will identify up to 6 key program staff and/or providers per site each year.

\section{Description of processes}

We will use a variety of data sources to compare the effectiveness of the two models including program inventories, surveys, interviews, focus groups, and patient physical measures (A1c, BMI, PHQ-9, and hair cortisol). Domains of inquiry include: $a$.) program accommodation of patients' language preference, cultural values, and socio-economic limitations, b.) program-related interpersonal interaction and communication, c.) program design to encourage social support among participants, d.) social "fit" with and social support from program peers, e.) social support from program staff, $f$.) diabetes health knowledge, g.) diabetes self-activation, $h$.) stress management, $i$.) A1c control, and $j$.) lifestyle changes to support diabetes health. Program assessment data will allow us to characterize the nature of each program and its approach to cultural competence. Survey responses and clinical test results from a large sample at each site will yield empirical (quantitative) data on patientreported outcomes. Interviews and focus groups with a subset of participants will provide rich, in-depth, contextually meaningful (qualitative) data regarding the domains of inquiry from the perspective of patients, social supports, and program staff/providers.

All study activities will be conducted in English or Spanish, depending on the language preference of the participant. For each component of the study, the participant will receive a $\$ 50$ merchandise card.

\section{Programmatic assessment}

We will inventory each program regarding program design, size, structure, operation, and theoretical/ 
philosophical orientation; professional qualifications/ training of program providers; activities or resources available through the programs; strategies in place for Spanish language use or acceptance, inclusion of social supports and family, accommodation of challenges created by patients' limited socioeconomic circumstances, and the inclusion of stress management techniques; and data on referrals to the program, sign-ups, participation, no-shows, and attrition. To gather qualitative information, we will conduct interviews with providers and staff at each site. We will assess patient and social support participants' perceptions of the program interface using a cultural competence survey $[19,20]$ that asks about physician bias, inter-cultural understanding, respectful interactions, language barriers, experiences of discrimination, and issues of trust. To gather qualitative information about cultural competence, we will include questions on programmatic interface in interviews and focus groups with patients and social supports (described below).

\section{Surveys}

We will hire four individuals from the population of study to work as Data Collectors. The Data Collectors will administer the survey orally to all patient and social support participants at baseline (when they enter the study), with follow-up at three, six, and 12 months. All participants will be asked to provide: 1.) Contact information to be used for follow-up, notification of high A1c results, and to invite the participant to the end-of-project presentation of data to study participants; and 2.) Demographic information to be used to characterize the participant and to be used as covariates in the proposed analyses. The survey will consist of questions from or modified from four tools validated and found to be reliable in our population:

1.) The Consumer Assessment of Healthcare Providers and Systems Cultural Competence Set (CAHPS-CC) $[19,20]$ [used as part of the programmatic assessment discussed in the preceding paragraph-will be administered at 3, 6 and 12 months only].

2.) The Diabetes Knowledge Questionnaire (DKQ) [21-23].

3.) The Patient Activation Measure (PAM-13) [24-32].

4.) The Patient Health Questionnaire 9 (PHQ-9) [33-36].

\section{Interviews}

We will conduct 72 interviews with patients and social supports (12 per year $\times 2$ sites $\times 3$ years). We believe that data from 12 interviews per site per year will capture a sufficient range of responses to achieve thematic saturation [37]. Interviews will last one to $2 \mathrm{~h}$ and will be semi-structured. We will audio-record interview sessions and recordings will be transcribed and translated if necessary.

\section{Focus groups}

We will conduct 12 focus groups $(2$ per year $\times 2$ sites $\times 3$ years). Focus group participants will be distinct from those recruited to participate in the interviews described in the preceding paragraph. Focus groups will include six distinct participants each. Focus groups will last one to $2 \mathrm{~h}$ and will follow a semi-structured focus group guide. We will audio-record focus group sessions and recordings will be transcribed and translated if necessary.

\section{Physical measures}

We will obtain the following measures from patients at baseline, 3, 6, and 12 months:

A1c The phlebotomy-trained Data Collector will draw blood samples at the same time they administer the survey. A1c will be tested on whole blood using the DCA Vantage 2000 analyzer.

Body mass index (BMI) Patient participants' BMI will be calculated using height and weight measurements obtained by the data collectors. Height and weight measurements will be taken using a standardized protocol. Two measurements will be taken for height and two for weight for each participant at each data collection point. An average of the two measures of height and an average of the two measures of weight will be used in the BMI calculation. The calculation will be done automatically in the database.

Hair cortisol To obtain objective information about the biological level of chronic stress, we will measure cortisol in hair. Hair cortisol is emerging as a reliable test for circulating levels of cortisol, which is an indication of chronic stress [38-40]. Hair will be gathered from patients only and not from social supports, and will only be gathered at two time points: baseline and 6 months (note: hair samples will only be gathered at baseline and 6 months).

\section{Database}

The data just described will be managed using REDCap software [41] which includes data capture, storage, and reporting capabilities. Surveys will be designed in REDCap to capture study participants' contact information, demographic information, and responses to the survey questions. The physical measures of patient participants will also be stored in the REDCap database, and recruitment, data collection appointments, and participation in qualitative data gathering activities will also be tracked using the software. 


\section{Statistical analysis \\ Hypothesized effect sizes and sample size requirements for outcomes}

We hypothesize that the CCM model will be superior to the DSMS in its ability to increase diabetes knowledge and patient activation, lower A1c, and improve depression scale scores, BMI, and levels of stress among participants. Our hypothesized effect sizes and measures of variability are based on previously reported improvements as measured by the various instruments (cited for each measure in Table 2).

\section{Sample size and power calculations}

We will recruit $N=240$ patient-social support pairs ( $n=120$ per site) with anticipated $20 \%$ attrition to obtain complete data on at least $n=96$ pairs per site. Table 2 describes power for hypothesized effect sizes between the CCM and DSMS between the change scores from baseline to 6 months on the DKQ, and PAM-13. Similarly, we report power for the secondary measures (A1c, BMI, and PHQ-9), as well. We assumed a two-sided type I error rate and adjusted for the number of comparisons made (two comparisons for the co-primary outcomes; three for the co-secondary outcomes) using a Bonferroni correction ( $\alpha=0.025, \alpha=0.017$, respectively). The power analyses for detecting site differences among change scores were based on multiple linear regression models including demographic characteristics, participants' perceived cultural competence of providers (CAHPS-CC), and social supports' change scores on the DKQ, PAM-13, and PHQ9 as covariates. We report Cohen's $f$ effect sizes based on the regression method $[42,43]$.

\section{Statistical analysis}

We will use descriptive statistics to summarize patient and social support characteristics. We will calculate means and standard deviations or medians and quartiles for continuous variables and will compare them across sites by $t$ test or Wilcoxon rank sum test, depending on the distribution of the data. We will calculate frequencies and percentages for categorical variables and will compare them with the chi-square test or Fisher's exact test, as appropriate. To assess how the primary and secondary outcome measures change over time, we will fit linear mixed models to each outcome with the primary independent variable in each model being treatment site. The models will include covariates including participant characteristics, measures of social support, and exploratory measure of hair cortisol. Interactions between time and model will be included in each mixed model to determine whether measures change differentially between the sites over time. To address potential heterogeneity of treatment effects for poverty status (less than FPL versus between FPL and 250\% FPL), we will also include an interaction term between poverty status and site. We will report adjusted least-squares mean difference estimates and appropriate confidence intervals of the outcome measures.

Table 2 Hypothesized effect sizes and measures of variability

\begin{tabular}{|c|c|c|c|c|c|c|}
\hline Outcomes & Measures & Method/Instrument & Hypothesis $^{a}$ & $\begin{array}{l}\text { Hypothesized } \\
\text { Change score, } \\
\Delta_{\text {DSCS-CCM }}(\mathrm{SD})\end{array}$ & $\begin{array}{l}\text { Hypothesized } \\
\text { Cohen's } f \\
\text { effect size }\end{array}$ & Power \\
\hline \multirow{2}{*}{$\begin{array}{l}\text { Primary Outcome: } \\
\text { Improved Capacity } \\
\text { for Diabetes Self- } \\
\text { Management }\end{array}$} & Diabetes Knowledge & DKQ summed score & $\begin{array}{l}\text { The CCM Model will result } \\
\text { in a larger increase in DKQ } \\
\text { summed scores }\end{array}$ & $\begin{array}{l}2.2(3.8) \\
\text { (references [21-23]) }\end{array}$ & 0.09 & $96 \%$ \\
\hline & Patient Activation & PAM-13 raw score & $\begin{array}{l}\text { The CCM model will result } \\
\text { in a larger increase in } \\
\text { PAM-13 raw scores }\end{array}$ & $\begin{array}{l}12.7(24.8) \\
\text { (references }[24-32,44])\end{array}$ & 0.07 & $85 \%$ \\
\hline \multirow[t]{3}{*}{$\begin{array}{l}\text { Secondary Outcome: } \\
\text { Successful Diabetes } \\
\text { Self-Management }\end{array}$} & AlC & $\% \mathrm{~A} 1 \mathrm{C}$ & $\begin{array}{l}\text { The CCM model will result } \\
\text { in a larger decrease in } \\
\text { percent } A 1 C\end{array}$ & $\begin{array}{l}-0.5(1.0) \\
\text { (references [45-48]) }\end{array}$ & 0.06 & $84 \%^{b}$ \\
\hline & $\mathrm{BMI}$ & $\mathrm{BMI}$ & $\begin{array}{l}\text { The CCM model will result } \\
\text { in a larger decrease in BMI }\end{array}$ & $\begin{array}{l}-1.5(3) \mathrm{kg} / \mathrm{m}^{2} \\
\text { (references }[47,49])\end{array}$ & 0.06 & $84 \%^{b}$ \\
\hline & Depression & $\begin{array}{l}\text { PHQ-9 summed } \\
\text { score }\end{array}$ & $\begin{array}{l}\text { The CCM model will result } \\
\text { in a larger decrease in PHQ- } \\
9 \text { summed scores }\end{array}$ & $\begin{array}{l}-3(6) \\
\text { (references [50-52]) }\end{array}$ & 0.06 & $84 \%^{b}$ \\
\hline $\begin{array}{l}\text { Exploratory Outcome: } \\
\text { Improved Stress } \\
\text { Management }\end{array}$ & Hair Cortisol & $\begin{array}{l}\text { Cortisol amount (pg/ } \\
\text { mg) }\end{array}$ & $\begin{array}{l}\text { The CCM model will result in } \\
\text { greater decrease in average } \\
\text { cortisol levels }\end{array}$ & $\begin{array}{l}4(6) \\
\text { (references [38-40, 53-58]) }\end{array}$ & 0.05 & $95 \%$ \\
\hline
\end{tabular}

${ }^{a}$ Changes over time are from baseline to 6 months

${ }^{b}$ It is coincidental that the power (and effect sizes) are the same for each of the three secondary outcomes $B M I$ body mass index

$D K Q$ diabetes knowledge questionnaire

$P A M-13$ patient activation measure 13-item instrument PHQ-9 patient health questionnaire 9-item instrument

$\mathrm{Pg} / \mathrm{mg}$ picogram of cortisol per milligram of hair 


\section{Qualitative analysis}

We will complement our quantitative analyses by conducting analyses of data from key staff/provider interviews (Aim \#1), and patient/social support interviews and focus groups (Aims \#1 \& 2). We will follow criteria for a theory-driven qualitative content analysis [43]. We will identify themes, and code and subcode transcripts to explore interconnections between theme categories and to develop a holistic interpretation of the data.

\section{Discussion}

It is common in studies of diabetes to have the primary outcome be A1c levels. But in this patient-engaged study, patients wanted us to take a different approach. We know how to get people to lower their A1c; the issue is getting them to do those things. Patients told us that you have to understand your diabetes, have the capacity to make changes in your life, and know how to control your stress before you can even think about lowering your A1c. Therefore, we said that "capacity" is measured by our two primary outcomes (diabetes knowledge and diabetes-related patient activation) and the success of the capacity is measured by the biological measures of A1c, BMI, depression, and cortisol. We hypothesize that the program that is most culturally and contextually situated will do the best job educating people about their diabetes in a meaningful, lasting way, helping them to become empowered (activated), and helping them or giving them strategies to deal with stress in their lives. Therefore the most culturally and contextually situated program will be the most "culturally competent" and will have the best impact on capacity for diabetes selfmanagement (as measured by diabetes knowledge and patient activation in relation to diabetes health). We also expect to see primary, secondary, and exploratory outcomes to be correlated-e.g., patients with better knowledge and activation are likely to have lower A1c levels, lower BMI, improved depression scores, and lowered cortisol levels.

In addition, patients told us that the common approach to measuring diabetes control at the individual patient level fails to appreciate the cultural and social context of people's lives, which they believe is essential for understanding diabetes self-management for Latinos. To attempt to capture this social dimension of people's lives, we are recruiting patients and social supports as dyads. Patient outcomes will be measured and information we gather from social supports will inform our understanding of those results.

Obstacles we anticipate include confounders that may influence statistical analyses, attrition in participation that may make data collection difficult, and identification of A1c and depression levels that could require that we refer patients for further care. At the end of this 3- year study we expect to be able to identify the characteristics of diabetes self-management programs that make them culturally competent.

\begin{abstract}
Abbreviations
A1c: HbA1c blood test for glycated hemoglobin or glycosylated hemoglobin to measure blood sugar level; BMI: Body mass index; CAHPS-CC: The Consumer Assessment of Healthcare Providers and Systems Cultural Competence Set; CCM: The chronic care model; DKQ: The diabetes knowledge questionnaire; DSMS: The Diabetes Self-Management Support Empowerment Model (DSMS); FPL: Federal poverty level; PAM-13: The patient activation measure (PAM-13; PCP: Primary care provider; $\mathrm{pg} / \mathrm{mg}$ : Picogram of cortisol per milligram of hair; PHQ-9: The patient health questionnaire 9; REDCap: Research Electronic Data Capture - a browser-based clinical and translational research database software program
\end{abstract}

\section{Acknowledgements}

We would like to recognize the contribution of our Patient Advisory Board members who met monthly over a period of 2 years to provide substantial input for the design of this patient-engaged study. We would also like to acknowledge Mary Lynn Johnston at the UNM Hospital Center for Diabetes Education, and Blanca Pedigo and John Bulten at One Hope Centro de Vida Health Center/East Central Ministries, who are our site partners. Hannah Cole-McGrew joined our team after the project was funded and is now providing significant support for implementation of the study, and Maria Tellez has been our patient engagement coordinator and now manages the activities of community data collectors, Virginia Sandoval, Denisse Guerrero, and Loida Varela.

\section{Funding}

The research described in this protocol has been funded through a peer review process by the Patient-Centered Outcomes Research Institute (PCORI) under \# CER-1511-32,910 and is currently in progress. The production of this article was supported in part by PCORI Awards (Tier I-\#7,738,704, Tier II-\#7,738,704, \& \#CER1511-32,910). The views presented in this work are solely the responsibility of the authors and do not necessarily represent the views of the PCORI and its Board of Governors or Methodology Committee. Additional personnel training and clinical laboratory expertise was provided by the University of New Mexico Clinical and Translational Research Center (NCATS \#8UL1TR000041).

\section{Availability of data and materials}

As this study just began to enroll participants in February 2017, no data is currently available to be shared. Following the end of the study, the de-identified datasets generated and/or analysed will be stored in the UNM data repository, LoboVault, a designated long-term digital archive resource maintained by the UNM Libraries. Quantitative data will be available from the corresponding author on reasonable request. Qualitative data (interview and focus group transcripts) will not be shared because this data will have been gathered from a small sample and will contain a large amount of personal information that would identify the participant.

\section{Authors' contributions}

JPR, the PI, brought together the team, conceptualized the study design, worked closely with the patient partner (Co-PI) to engage patient stakeholders to develop the research question and patient-identified outcome measures, was the lead writer, and wrote sections on program inventory, interviews, focus groups, qualitative analysis and patient characteristics. $L R$, the patient partner Co-PI, convened a Patient Advisory Board, worked closely with the PI to engage patient stakeholders as indicated above, and provided input to ensure the cultural appropriateness of the project design and data collection techniques. CMK was the principal designer of the quantitative data analysis plan and primary author of sections regarding power, sample size, and analysis of outcome data. $\mathrm{MB}$ identified the database software and the instruments and wrote sections describing the survey psychometrics and use, wrote sections describing data management and storage, and assisted with bibliographic materials. EE was a major contributor in the design and writing of the quantitative data analysis plan. MRB designed the component of the study involving analysis of patient A1c data and advised on study design related to participant eligibility, crisis management planning for patients found to have high A1c, and all aspects of diabetes health that needed to be considered in the design and implementation of this study. ELB designed the component of the study involving analysis of patient hair samples for circulating levels of cortisol as a biomarker for 
chronic stress. She provided language regarding hair collection, analysis, and storage procedures. She reviewed and edited the MS and drafted the discussion. SIM provided guidance for incorporating patient-identified outcome measures into the study design and was a major contributor in writing the manuscript. All authors read and approved the final manuscript.

\section{Ethics approval and consent to participate}

This study was approved by the Human Research Protections Office (HRPO) which is the ethnics committee for the University of New Mexico (UNM) on October 7, 2016 (HRPO study approval \#16-303). The study began recruiting in February 2017. All participants will provide written informed consent.

\section{Consent for publication}

Not applicable.

\section{Competing interests}

The authors declare that they have no competing interests.

\section{Publisher's Note}

Springer Nature remains neutral with regard to jurisdictional claims in published maps and institutional affiliations.

\section{Received: 11 May 2017 Accepted: 5 July 2017}

Published online: 24 July 2017

\section{References}

1. Beckles G, Zhu J, Moonesinghe R. Diabetes - United States, 2004 and 2008 Morb Mortal Wkly Rep. 2011;60(Suppl 1):90-3.

2. Barrera M, Castro FG, Strycker LA, Toobert DJ. Cultural adaptations of behavioral health interventions: A progress report. J Consult Clin Psychol. 2013;81(2):196-205. doi:10.1037/a0027085.

3. Hawthorne K, Robles Y, Cannings-John R, Edwards AGK. Culturally appropriate health education for Type 2 diabetes in ethnic minority groups: a systematic and narrative review of randomized controlled trials. Diabet Med J Br Diabet Assoc. 2010;27(6):613-23. doi:10.1111/j.1464-5491. 2010.02954.x.

4. Kong A, Tussing-Humphreys LM, Odoms-Young AM, Stolley MR, Fitzgibbon ML. Systematic review of behavioural interventions with culturally adapted strategies to improve diet and weight outcomes in African American women. Obes Rev Off J Int Assoc Study Obes. 2014; 15(Suppl 4):62-92. doi:10.1111/obr.12203.

5. Lie DA, Lee-Rey E, Gomez A, Bereknyei S, Braddock CH. Does cultural competency training of health professionals improve patient outcomes? A systematic review and proposed algorithm for future research. J Gen Intern Med. 2011;26(3):317-25. doi:10.1007/s11606-010-1529-0.

6. Nam S, Janson SL, Stotts NA, Chesla C, Kroon L. Effect of culturally tailored diabetes education in ethnic minorities with type 2 diabetes: a metaanalysis. J Cardiovasc Nurs. 2012;27(6):505-18. doi:10.1097/JCN. Ob013e31822375a5.

7. Pottie K, Hadi A, Chen J, Welch V, Hawthorne K. Realist review to understand the efficacy of culturally appropriate diabetes education programmes. Diabet Med J Br Diabet Assoc. 2013;30(9):1017-25. doi:10. 1111/dme.12188.

8. Ricci-Cabello I, Ruiz-Pérez I, Rojas-García A, Pastor G, Rodríguez-Barranco M Gonçalves DC. Characteristics and effectiveness of diabetes selfmanagement educational programs targeted to racial/ethnic minority groups: a systematic review, meta-analysis and meta-regression. BMC Endocr Disord. 2014;14:60. doi:10.1186/1472-6823-14-60.

9. Whittemore R. Culturally competent interventions for Hispanic adults with type 2 diabetes: a systematic review. J Transcult Nurs Off J Transcult Nurs Soc Transcult Nurs Soc. 2007;18(2):157-66. doi:10.1177/1043659606298615.

10. Zeh P, Sandhu HK, Cannaby AM, Sturt JA. The impact of culturally competent diabetes care interventions for improving diabetes-related outcomes in ethnic minority groups: a systematic review. Diabet Med J Br Diabet Assoc. 2012;29(10):1237-52. doi:10.1111/j.1464-5491.2012.03701.x.

11. Dauvrin $\mathrm{M}$, Lorant $\mathrm{V}$, $\mathrm{d}^{\prime}$ Hoore $\mathrm{W}$. Is the chronic care model integrated into research examining culturally competent interventions for ethnically diverse adults with Type 2 diabetes mellitus? A review. Eval Health Prof. 2015. doi: $10.1177 / 0163278715571004$.
12. Brown A, Lopez MH. Ranking Latino Populations in the States. Pew Res Cent Hisp Trends Proj. August 2013. http://www.pewhispanic.org/2013/08/29/iiranking-latino-populations-in-the-states/. Accessed 2 Feb 2016.

13. United States Census Bureau. American Community Survey Demographics and Housing Estimates 2012. American Fact Finder. https://factfinder.census. gov/faces/tableservices/jsf/pages/productview.xhtml?src=bkmk. Accessed 1 Sept 2014.

14. The Heller School for Social Policy and Management. Albuquerque, NM diversitydata.org. http://www.diversitydata.org/Data/Profiles/Show.aspx?loc=68. Accessed 4 Feb 2016

15. New Mexico Department of Health. New Mexico's Indicator-Based Information System. https://ibis.health.state.nm.us/. Accessed 4 Feb 2016.

16. Ennis SR, Rios-Vargas M, Albert NG. The Hispanic Population: 2010. United States Census Bureau; 2011. http://www.census.gov/prod/cen2010/briefs/ c2010br-04.pdf. Accessed 2 Feb 2016.

17. Funnell MM, Brown TL, Childs BP, Haas LB, Hosey GM, Jensen B, Maryniuk M, Peyrot M, Piette JD, Reader D, Siminerio LM, Weinger K, Weiss MA. National Standards for diabetes self-management education. Diabetes Care. 2011; 34(Suppl 1):S89-96. doi:10.2337/dc11-S089.

18. Stellefson M, Dipnarine K, Stopka C. The chronic care model and diabetes management in US primary care settings: a systematic review. Prev Chronic Dis. 2013;10:E26. doi:10.5888/pcd10.120180.

19. Agency for Healthcare Research and Quality. CAHPS: Assessing Health Care Quality From the Patient's Perspective. US Department of Health and Human Services; 2014. https://www.ahrq.gov/cahps/about-cahps/cahpsprogram/cahps_brief.html. Accessed 10 Nov 2015.

20. Agency for Healthcare Research and Quality. About the CAHPS Cultural Competence Item Set. https://cahps.ahrq.gov/surveys-guidance/survey4.0docs/2312_about_cultural_comp.pdf. Accessed 10 Nov 2015.

21. Sixta CS, Ostwald S. Texas-Mexico border intervention by promotores for patients with type 2 diabetes. Diabetes Educ. 2008;34(2):299-309. doi:10. $1177 / 0145721708314490$.

22. Mauldon M, Melkus GD, Cagganello M. Tomando Control: a culturally appropriate diabetes education program for Spanish-speaking individuals with type 2 diabetes mellitus-evaluation of a pilot project. Diabetes Educ. 2006:32(5):751-60. doi:10.1177/0145721706291999.

23. Garcia AA, Villagomez ET, Brown SA, Kouzekanani K, Hanis CL. The Starr County Diabetes Education Study: development of the Spanish-language diabetes knowledge questionnaire. Diabetes Care. 2001;24(1):16-21.

24. Schiøtz ML, Bøgelund M, Almdal T, Jensen BB, Willaing I. Social support and self-management behaviour among patients with Type 2 diabetes. Diabet Med J Br Diabet Assoc. 2012;29(5):654-61. doi:10.1111/j.1464-5491.2011.03485.x.

25. Rygg $L \varnothing$, Rise MB, Grønning K, Steinsbekk A. Efficacy of ongoing group based diabetes self-management education for patients with type 2 diabetes mellitus. A randomised controlled trial. Patient Educ Couns. 2012 86(1):98-105. doi:10.1016/j.pec.2011.04.008.

26. Parchman ML, Zeber JE, Palmer RF. Participatory decision making, patient activation, medication adherence, and intermediate clinical outcomes in type 2 diabetes: a STARNet study. Ann Fam Med. 2010;8(5):410-7. doi:10. 1370/afm.1161.

27. Hibbard JH, Mahoney ER, Stock R, Tusler M. Do increases in patient activation result in improved self-management behaviors? Health Serv Res. 2007:42(4):1443-63. doi:10.1111/j.1475-6773.2006.00669.x.

28. Hibbard JH, Mahoney E. Toward a theory of patient and consumer activation. Patient Educ Couns. 2010:78(3):377-81. doi:10.1016/j.pec.2009.12.015.

29. Hibbard JH. Community-based participation approaches and individual health activation. J Ambulatory Care Manage. 2009:32(4):275-7. doi:10.1097/ JAC.0b013e3181ba6f63.

30. Hendriks M, Rademakers J. Relationships between patient activation, disease-specific knowledge and health outcomes among people with diabetes; a survey study. BMC Health Serv Res. 2014;14:393. doi:10.1186/ 1472-6963-14-393.

31. Dixon A, Hibbard J, Tusler M. How do People with Different Levels of Activation Self-Manage their Chronic Conditions? The Patient. 2009;2(4):25768. doi:10.2165/11313790-000000000-00000.

32. Hibbard JH, Stockard J, Mahoney ER, Tusler M. Development of the Patient Activation Measure (PAM): conceptualizing and measuring activation in patients and consumers. Health Serv Res. 2004;39(4 Pt 1):1005-26. doi:10. 1111/j.1475-6773.2004.00269.x.

33. Huang FY, Chung H, Kroenke K, Delucchi KL, Spitzer RL. Using the Patient Health Questionnaire-9 to measure depression among racially and 
ethnically diverse primary care patients. J Gen Intern Med. 2006;21(6):54752. doi:10.1111/j.1525-1497.2006.00409.x.

34. Gillbody S, Richards D, Brealey S, Hewitt C. Screening for depression in medical settings with the Patient Health Questionnaire (PHQ): a diagnostic meta-analysis. J Gen Intern Med. 2007;22(11):1596-602. doi:10.1007/s11606007-0333-y.

35. Reuland DS, Cherrington A, Watkins GS, Bradford DW, Blanco RA, Gaynes BN. Diagnostic accuracy of Spanish language depression-screening instruments. Ann Fam Med. 2009; 7(5):455-62. doi:10.1370/afm.981.

36. Kroenke K, Spitzer RL, Williams JBW. The PHQ-9: Validity of a Brief Depression Severity Measure. J Gen Intern Med. 2001;16(9):606-13. doi:10. 1046/j.1525-1497.2001.016009606.x.

37. Yamada J, Stevens B, de Silva N, Gibbins S, Beyene J, Taddio A, Newman C, Koren $\mathrm{G}$. Hair cortisol as a potential biologic marker of chronic stress in hospitalized neonates. Neonatology. 2007:92(1):42-9. doi:10.1159/ 000100085 .

38. Guest G, Bunce A, Johnson L. How Many Interviews Are Enough? Field Methods. 2006;18(1):59-82.

39. Lehrer HM, Dubois SK, Maslowsky J, Laudenslager ML, Steinhardt MA. Hair cortisol concentration and glycated hemoglobin in African American adults. Psychoneuroendocrinology. 2016;72:212-8. doi:10.1016/j. psyneuen.2016.06.018.

40. Larsen SC, Fahrenkrug J, Olsen NJ, Heitmann BL. Association between Hair Cortisol Concentration and Adiposity Measures among Children and Parents from the "Healthy Start" Study. PLoS One. 2016;11(9):e0163639. doi:10.1371/ journal.pone.0163639.

41. Kim S, Love F, Quistberg DA, Shea JA. Association of health literacy with self-management behavior in patients with diabetes. Diabetes Care. 2004; 27(12):2980-2.

42. Selya AS, Rose JS, Dierker LC, Hedeker D, Mermelstein RJ. A Practical Guide to Calculating Cohen's $\mathrm{f}(2)$, a Measure of Local Effect Size, from PROC MIXED. Front Psychol. 2012;3:111. do:10.3389/fpsyg.2012.00111.

43. Gläser J, Laudel G. Life with and without coding: Two methods for earlystage data analysis in qualitative research aiming at causal explanations. Forum Qual Sozialforschung. 2013;14(2). http://www.qualitative-research.net/ index.php/fas/article/view/1886.

44. Wolever RQ, Dreusicke M, Fikkan J, Hawkins TV, Yeung S, Wakefield J, Duda L, Flowers P, Cook C, Skinner E. Integrative health coaching for patients with type 2 diabetes: a randomized clinical trial. Diabetes Educ. 2010;36(4):629-39. doi:10. 1177/0145721710371523.

45. Druss BG, Zhao L, von Esenwein SA, Bona JR, Fricks L, Jenkins-Tucker S, Sterling E, DiClemente R, Lorig K. The Health and Recovery Peer (HARP) Program: A peer-led intervention to improve medical self-management for persons with serious mental illness. Schizophr Res. 2010;118(1-3):264-70. doi:10.1016/j.schres.2010.01.026.

46. Grønning K, Rannestad T, Skomsvoll JF, Rygg LØ, Steinsbekk A. Long-term effects of a nurse-led group and individual patient education programme for patients with chronic inflammatory polyarthritis - a randomised controlled trial. J Clin Nurs. 2014;23(7-8):1005-17. doi:10.1111/jocn.12353.

47. Lorig K, Ritter PL, Villa FJ, Armas J. Community-based peer-led diabetes selfmanagement: a randomized trial. Diabetes Educ. 2009;35(4):641-51. doi:10. 1177/0145721709335006.

48. Looker HC, Knowler WC, Hanson RL. Changes in BMl and weight before and after the development of type 2 diabetes. Diabetes Care. 2001;24(11):1917-22.

49. Avery L, Flynn D, van Wersch A, Sniehotta FF, Trenell MI. Changing physical activity behavior in type 2 diabetes: a systematic review and meta-analysis of behavioral interventions. Diabetes Care. 2012;35(12):2681-9. doi:10.2337/ dc11-2452.

50. Bogner HR, Morales KH, de Vries HF, Cappola AR. Integrated management of type 2 diabetes mellitus and depression treatment to improve medication adherence: a randomized controlled trial. Ann Fam Med. 2012; 10(1):15-22. doi:10.1370/afm.1344.

51. Lorig K, Ritter PL, Laurent DD, Plant K, Green M, Jernigan VBB, Case S. Online diabetes self-management program: a randomized study. Diabetes Care. 2010;33(6):1275-81. doi:10.2337/dc09-2153.

52. Sauvé B, Koren G, Walsh G, Tokmakejian S, Van Uum SH. Measurement of cortisol in human hair as a biomarker of systemic exposure. Clin Invest Med. 2007;30(5):E183-91. PMID: 17892760

53. Russell E, Kirschbaum C, Laudenslager ML, Stalder T, de Rijke Y, van Rossum EF, Van Uum S, Koren G. Toward standardization of hair cortisol measurement: results of the first international interlaboratory round robin. Ther Drug Monit. 2015;37(1):71-5. doi:10.1097/FTD. 0000000000000148. PMID: 253872

54. Staufenbiel SM, Penninx BW, de Rijke YB, van den Akker EL, van Rossum EF. Determinants of hair cortisol and hair cortisone concentrations in adults. Psychoneuroendocrinology. 2015;60:182-94. doi:10.1016/j.psyneuen.2015.06. 011. PMID: 26176863

55. Short SJ, Stalder T, Marceau K, Entringer S, Moog NK, Shirtcliff EA, Wadhwa PD, Buss C. Correspondence between hair cortisol concentrations and 30-day integrated daily salivary and weekly urinary cortisol measures. Psychoneuroendocrinology. 2016;71:12-8. doi:10.1016/j.psyneuen.2016.05.007.

56. Ursache A, Merz EC, Melvin S, Meyer J, Noble KG. Socioeconomic status, hair cortisol and internalizing symptoms in parents and children. Psychoneuroendocrinology. 2017;78:142-50. doi:10.1016/.jpsyneuen.2017.01. 020. [Epub ahead of print]. PMID: 28199857

57. Aguiló J, Ferrer-Salvans P, García-Rozo A, Armario A, Corbí Á, Cambra FJ, Bailón R, González-Marcos A, Caja G, Aguiló S, López-Antón R, Arza-Valdés A, Garzón-Rey JM. Project ES3: attempting to quantify and measure the level of stress. Rev Neurol. 2015;61(9):405-15. PMID: 26503316

58. Cohen JE. Statistical Power Analysis for the Behavioral Sciences. Lawrence Erlbaum Associates, Inc: Hillsdale; 1988.

\section{Submit your next manuscript to BioMed Central and we will help you at every step:}

- We accept pre-submission inquiries

- Our selector tool helps you to find the most relevant journal

- We provide round the clock customer support

- Convenient online submission

- Thorough peer review

- Inclusion in PubMed and all major indexing services

- Maximum visibility for your research

Submit your manuscript at www.biomedcentral.com/submit
C) Biomed Central 